\title{
CARACTERIZACIÓN DE LA FRACCIÓN LIPIDICA DE TRES RAZAS DE PIJUAYO (Bactris gasipaes H.B.K)
}

\author{
Dora E. Garcia * \\ Victror E. Sotero* \\ Edson Lessi**
}

\section{RESUMEN}

En el presente estudio se caracterizó el fruto y la fracción lipídica del mesocarpio de tres razas de pijuayo: «Microcarpa» Pará, «Mesocarpa» Solimóes y «Macrocarpa» Putumayo.

Los frutos fueron colectados del Banco Activo de Germoplasma de Pijuayo del Instituto Nacional de Pesquisas de la Amazonia (INPA)-Manaus, Brasil. Los aceites del mesocarpio de cada muestra frieron extraídos con el aparato de Soxhlet, y la composición de los ácidos grasos, determinados por cromatografia gaseosa, fue principalmente de ácido palmítico, oleico y linoleico. Los tres grupos raciales no presentaron variación significativa en la concentración de palmítico y oleico, pero sí difieren significativamente en linoleico. Las concentraciones para la raza «Microcarpa» Pará fue de 49,7 y 5,9\% de ácido oleico y linoleico respectivamente; 42,3 y 12,3\% en la raza «Mesocarpa» Solimóes; 41,6 y 14,2\% en la raza «Macrocarpa» Putumayo.

\footnotetext{
ABSTRACT

Fmit and the mesocarp lipid fraction were characterized in samples from three different Peach Palm landraces: «Microcarpa» Pará, «Mesocarpa» Solimóes and «Macrocarpa» Putumayo.

This fi-uit were obtained from the INPA Pejibaye Gennplasma Bank at Manaus, Amazonas, Brazil. Oils from the mesocarp of evency landrace was extracted by the Soxhlet apparatus.

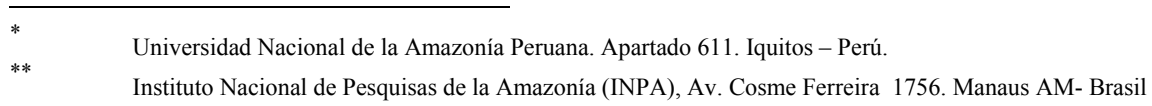


The lipid fractions were composed principally of palmitic, oleic and linoleic acid, with the palmitic relatively constant in the three landraces and the linoleic showing significant differences among the landraces 49,7 and 5,9\% in the Pará landrace for oleic and linoleic, respectively; $42,3 \%$ and $12,3 \%$ in the Solimóes; and 41,6 and $14,2 \%$ in the Putumayo.

Palabras clave: Aceite, ácidos grasos, Bactris gas ipaes, pijuayo, pejibaye, chontaduro.

\section{INTRODUCCION}

El pijuayo (Bactris gaszpaes H.B.K.) presenta una apreciable producción de frutos por hectárea y podria convenirse en un importante recurso para los paises del trópico húmedo como productor de los aceites y harina para la alimentación humana y/o animal; además se utiliza el corazón del tallo en la preparación de palmito (Villachica, 1996).

Mora Urpi \& Clement (1985) propusieron la clasificación de los grupos raciales de pijuayo de la cuenca amazónica basados en el tamaño del fruto, distribuidos según la siguiente ubicación:

Microcarpa. Con frutos pequeños, son los más primitivos, siendo poco modificados a partir de poblaciones salvajes tales como la Guilielrna microcaipa; aquí se colocan a las razas Juruá y Pará (Brasil).

Mesocarpa. Fue completamente domesticada y está compuesta por las razas

Pastasa (Ecuador), Pampa Hermosa (Perú), Solimóes (Brasil) e Inirida (Colombia).

Macrocarpa. Con los frutos mayores, producto de la mejor selección y domesticación, contiene a las razas Putumayo (Perú) y Vaupés (Colombia / Brasil). La literatura presenta una amplia variación, en los valores encontrados en los análisis químicos realizados por los investigadores; sin embargo, todos le atribuyen un alto valor nutritivo. Aguiar et al., (1980) encontraron una concentración de $27 \%$ de aceite, $3,5 \%$ de proteínas, $0,9 \%$ de cenizas $3,8 \%$ de fibra y $23,6 \%$ de carbohidratos, además de una elevada concentración de vitamina «A». El aceite

De pijuayo merece ser destacado como un componente de importante valor económico (Clement \& Mora Urpi, 1984). 
Arkcool \& Aguiar (1984) encontraron frutos con concentraciones de $62 \%$ de aceite en el mesocarpio. Clernent \& Arkcool, en Clement (1993), indicaron posteriormente que los frutos más aceitosos son las poblaciones del grupo racial «rnicrocarpa».

Aunque el aceite haya sido una cualidad atractiva para los amerindios, parece ser que ellos no realizaron la selección buscando esa cualidad, sino más bien para la obtención de harina (Clement, 1988).

Serruya et al. (1980) trabajando con frutos de población no identificada, concluyen que el rendimiento en el aceite de pijuayo, especialmente de la pulpa, varia bastante, lo que sugiere un estudio más esmerado en el sentido de tentar descubrir los factores que determinan esta variación. El aceite de la almendra del pijuayo es semejante a los aceites de otras excepto por presentar un porcentaje de ácido láurico, superior a los demás.

Harnmond et al. (1982) y Kroneberg (1982) afirman que la composición cualitativa del aceite de pijuayo es similar a la encontrada en la palma africana o dendé, pero difiere fundamentalmente en el elevado porcentaje de ácido oleico, el cual es menos saturado y básicamente liquido a la temperatura ambiente -lo que le confiere una ventaja como producto alimenticio-, y la semilla presenta una mayor concentración de ácido láurico.

Zapata (1972) identificó cinco ácidos grasos en el fruto: palmítico, palmitoleico, esteárico, oleico y linoleico.

Zumbado y Murillo (1983) afirman que la mayoría de los ácidos grasos se presentan como ácidos grasos libres en una proporción equivalente a 2:1 de insaturados: saturados. Los ácidos grasos predominantes en el aceite de pijuayo son el ácido oleico y palmítico con menores niveles de ácido linoleico.

Gómez Da Silva \& Amelotti (1983) trabajando con pijuayo de población desconocida, encontraron que la concentración de ácido palmítico y oleico es mucho más abundante que el palmitoleico y linoleico.

En el Cuadro $\mathrm{N}^{\circ} 1$ se presentan las composiciones porcentuales de ácidos grasos en el aceite del mesocarpio de pijuayo, determinadas por cromatografia gaseosa, obtenidas por varios investigadores sin declarar las razas, y además la composición del aceite de la palma aceitera (Eleais ginensis) (Noiret \& Wuidart, en Clement, 1993). 
Cuadro 1. Composición porcentual de los ácidos grasos en el aceite del mesocarpio de los frutos del pijuayo (Bactris gasipaes H.B.K.).

\begin{tabular}{|l|l|l|l|l|l|l|l|l|}
\hline INVESTIGADOS & $\mathbf{1 4 : 0}$ & $\mathbf{1 5 : 0}$ & $\mathbf{1 6 : 0}$ & $\mathbf{1 6 : 1}$ & $\mathbf{1 8 : 0}$ & $\mathbf{1 8 : 1}$ & $\mathbf{1 8 : 2}$ & $\mathbf{1 8 : 3}$ \\
\hline $\begin{array}{l}\text { Hammond et al. (1982), } \\
\text { Cost.Ri. }\end{array}$ & - & - & 29,6 & 5,3 & Tra. & 50,3 & 12,3 & 1,8 \\
$\begin{array}{l}\text { Serruya et al. (1980), } \\
\text { Amazonia. }\end{array}$ & - & - & 40,2 & - & - & 53,6 & 6,3 & - \\
$\begin{array}{l}\text { Gómez Da Silva \& } \\
\text { Amelotti (1983), } \\
\text { Amazonia. } \\
\begin{array}{l}\text { Zumbado y Murillo } \\
\text { (1983), Costa Rica } \\
\text { Ciprona (1986), Cost. }\end{array}\end{array}$ & 0,2 & 0,1 & 44,8 & 6,5 & 1,5 & 41,0 & 4,8 & 1,0 \\
& - & - & 43,7 & 11,7 & 0,4 & 42,7 & 1,5 & - \\
\hline
\end{tabular}

\section{MATERIAL Y METODOS}

Los frutos del pijuayo de las tres razas fueron colectados de los árboles del Banco Activo de Germoplasma (BAG) de Pijuayo de la Estación Experimental de Fruticultura del Instituto Nacional de Pesquisas de la Amazonia (INPA), localizado en el Municipio de Manaos, AM Brasil.

La selección de los árboles para la colecta fue realizada al azar, con 10 palmeras de cada raza en estudio: «Microcarpa» Pará, «Mesocarpa» Solimóes y «Macrocarpa» Putumayo. Las colectadas de las zafras fueron realizadas en los periodos de octubre del año 1987 a febrero de 1988 y noviembre de 1988 a febrero de 1989

\subsection{Métodos}

\subsubsection{Análisis de la composición química}

El mesocarpio fue secado en estufa de aire circulante a $70^{\circ} \mathrm{C}$ y triturado en molino universal. La harina así obtenida fue mantenida en sacos de

polietileno cerrados a vació y almacenados a temperatura de $20^{\circ} \pm .1^{\circ} \mathrm{C}$. Fueron realizadas las determinaciones de grasa, proteína, ceniza y fibra según las Normas Analíticas del Instituto Adolfo Lutz (1985). La fracción nifext fue determinada por diferencia. 


\subsubsection{Extracción de aceite para análisis}

La extracción de aceite fue realizada sobre la harina seca, con auxilio del aparato de Soxhlet, con éter etílico. La fracción etérea obtenida fue colocada en frasco ámbar de boca ancha y tapado con papel aluminio perforado, para así permitir la evaporación espontánea del solvente.

\subsubsection{Determinaciones físicas y físico-químicas sobre el aceite del mesocarpio}

Se realizó las siguientes determinaciones: índice de refracción, índice de acidez, índice de saponificación e índice de yodo, según las Normas Analíticas del Instituto Adolfo Lutz (1985) y la densidad y material insaponificable según AOAC (1975).

\subsubsection{Determinación de la composición de los ácidos grasos de aceite del mesocarpio}

Las muestras de aceite de las tres razas de pijuayo fueron saponificadas y, en seguida, metiladas por el método del trifluoruro de Boro, según AOAC (1975). Las muestras así obtenidas fueron analizadas en un cromatógrafo de gas, marca Carlo Erba 3160, con detector de ionización de llama, usando una columna capilar de sílica fundida, de 10 metros de longitud y 0,25 de diámetro interior, conteniendo una película de SE 54 de $0,25 \mathrm{~cm}$ de espesor. El nitrógeno fue usado como gas de arrastre, regulado para proveer una velocidad lineal de $33 \mathrm{mt} / \mathrm{seg}$. (medidos a $150^{\circ} \mathrm{C}$ ) y flujo compatible con la relación $20: 1$. Inyección de $2 \mu 1$ de la solución aceite: $n$-hexano en la proporción 1:1, seguida de un intervalo de 30 segundos para la recuperación de la columna. La inyección fue hecha con la temperatura de horno a $50^{\circ} \mathrm{C}$ y después de 30 minutos iniciales, la temperatura fue programada linealmente a $6^{\circ} \mathrm{C} / \mathrm{min}$., hasta $280^{\circ} \mathrm{C}$. En estas condiciones, los ácidos grasos emergieron de la columna, solamente en el rango de 150 a $200^{\circ}$.

El registrador fue programado para una velocidad de papel de $40 \mathrm{~cm} /$ hora. La identificación de los ácidos grasos fue hecha basada en el tiempo de retención, comparando con las substancias patrones y finalmente se realizó una co-inyección. 


\subsubsection{Análisis estadísticos}

Los datos de las medidas físicas, de las determinaciones físico-químicas, de la composición química y la composición de los ácidos grasos fueron sometidos al análisis de varianza con la utilización de la prueba «Fx», de distribución estadística, en un nivel de significancia de 1 y $5 \%$ según Zar (1984).

\section{RESULTADOS}

\subsection{Análisis de la composición química}

Los resultados de los análisis de la composición química de los mesocarpios del fruto en base húmeda se presentan en la Tabla $\mathrm{N}^{\circ} 1$ y en base seca en la Tabla $\mathrm{N}^{\mathrm{o}}$ 2; dichas tablas presentan también varianza de las medias, de las diferentes determinaciones químicas de las tres razas en estudio, por la prueba de Tuckey en el nivel de $5 \%$.

\subsubsection{Análisis físico y físico-químico del aceite}

En la Tabla $\mathrm{N}^{\mathrm{o}} 3$ son presentados los resultados físicos y físico-químicos del aceite del mesocarpio seco del pijuayo, zafras de 1987 y 1988, con el análisis de varianza de las medidas de las diferentes determinaciones de las razas en estudio, comprobado por la prueba de Tuckey en el nivel de 500 .

\subsubsection{Análisis de la composición de los ácidos grasos de los aceites}

En la Tabla $\mathrm{N}^{\mathrm{o}} 4$ son presentados los resultados del análisis de la composición de los ácidos grasos de los aceites de los mesocarpios de los frutos del pijuayo, de las razas en estudio, comparados con la prueba de Tuckey en el nivel de 5\%.

\section{DISCUSION}

De acuerdo con los resultados de la Tabla $\mathrm{N}^{\mathrm{o}} 2$, se puede deducir lo siguiente:

La mayor concentración de aceite fue encontrada en la raza «Microcarpa» Pará $\mathbf{( 1 8 , 6 \%}$ en peso seco), valor superior a los obtenidos por Calzada (1986), quien encontró 8,27\% o con muestras de Costa Rica, y Aguiar et al. (1980), trabajando con muestras de los alrededores de Manaos, encontró un valor de $27 \%$ en peso 
seco; estos valores son aun menores al reportado por Arkcool \& Aguiar (1984) que encontraron frutos con $\mathbf{6 2} \%$ de aceite.

Las medidas de concentración de proteína varían poco, de 4,13\% de la Putumayo a $5,53 \%$ de la Solimôes, concentraciones muy superiores a las de Aguiar et al. (1980), que obtuvieron 3,5\%.

El peso de la fibra varió de 1,190 o para la raza Pará, hasta 2,5 60 o para la raza Solimôes. Estos valores son menores que los presentados por Aguiar (Op. cit.): $3,8 \%$.

Las concentraciones de sales minerales encontradas son notoriamente mayores en la raza «Microcarpa» Pará y «Mesocarpa» Solimôes (2,02 2,06), y también superiores al valor de $0,9 \%$ encontrado por Aguiar et al. (Op. cit.).

Apoyados en los resultados de la composición química, quedó caracterizado que la raza «Microcarpa» Pará puede ser considerada rica en aceite, en cuanto que las razas «Mesocarpa» Solimôes y «Macrocarpa» Putumayo son amiláceas.

De los análisis físicos y físico-químicos de los aceites de las muestras de las tres razas, las determinaciones que presentaron variaciones significativas entre razas fueron el índice de acidez, índice de yodo y materia insaponificable.

Los que no presentaron variación significativa fueron el índice de refracción, la densidad y el índice de saponificación (Tabla $\mathrm{N}^{\mathrm{o}} 3$ ).

Los valores obtenidos para el índice de refracción fueron de 1,47 para la raza «Microcarpa» Pará y «Mesocarpa» Solimôes y de 1,48 para la raza «Macrocarpa» Putumayo, valores mayores a los encontrados por el Ciprona $(1986)(1,45)$ en Costa

Rica y Serruya et al. (1980) $(1,45)$, con poblaciones brasileñas no identificadas. Los valores de la densidad de 0,91 para la raza «Microcarpa» Pará y 0,90 para las razas «Mesocarpa» Solimôes y «Macrocarpa» Putumayo, son muy semejantes a los encontrados por el Ciprona (Op. cit.) $(0,90)$ y Serruya et al. (Op. cit.).

La raza «Microcarpa» Pará presenta mayor índice de acidez (75,38 ó 37,84 expresado en ácido oleico \% p/p) y la raza «Mesocarpa» Solirnôes presenta el valor mayor $(121,14$ ó 60,81 expresado en ácido oleico \% p/p), quedando la raza «Macrocarpa» Putumayo con el 
valor intermedio ( 81,95 ó 40,60 expresado en ácido oleico \% p/p). Serruya et al.(Op. cit.) encontró índice de acidez de 46,70, valor inferior a los obtenidos en el presente trabajo.

En relación al índice de saponificación, se obtuvo para la raza «Microcarpa» Pará, 195,55; para la «Mesocarpa» Solimóes, 198,1100; y para la «Macrocarpa» Putumayo, 210,06.

El Ciprona (1986) reporta el valor de 192,0 y Serruya et al. obtienen 197,4.

En la determinación del índice del yodo se obtuvo 68,78, 72,89 y 59,24 para las razas «Microcarpa» Pará, «Mesocarpa» Solimóes y «Macrocarpa» Putumayo, respectivamente. Valores menores al encontrado por el Ciprona (Op. cit.) $(124,0)$.

En cuanto al material insaponificable, se obtuvo los valores de $1,02 \%$ para la raza «Microcarpa» Paráy 0,55\% para las otras dos razas «Mesocarpa» Solimóes y «Macrocarpa» Putumayo.

Este último valor 0,55\% es semejante al encontrado por Gómez Da Silva \& Amelotti (1983), de con poblaciones brasileñas no identificadas.

Los resultados de composición de los ácidos grasos, presentados en la Tabla $\mathrm{N}^{\circ} 4$, muestran que el ácido palmítico está presente en los aceites de las tres razas, con valores de 33,$19 ; 36,45$ y $35,12 \%$, respectivamente, valores muy próximos al pre- 2 sentado por el Ciprona (Op. cit.), de $38,3 \%$.

Gómez Da Silva \& Amelotti (1983) encontraron 44,8\%, posiblemente con muestras del Municipio de Manaos. Serruya et al. (1980), encontraron el valor de 40,17\% de ácido palmítico, sin especificar la presencia de la muestra.

Los valores de ácido oleico, obtenidos para las tres razas fueron 49,68\% para «Microcarpa» Pará; 42,30\% pal-a «Mesocarpa» Solimóes y 41,61\% para «Macrocarpa» Putumayo.

La literatura presenta valores diversos para el aceite de Pijuayo de razas no detenninadas: 50,3\% fueron obtenidas por Serruya et al. (Op. cit.); 50,3\%.

por Hammond et al. (1982); 41,\% por Gómez Da Silva \& Amelotti (Op. cit.) y 37,1\% por el Ciprona (Op. eit.). 
Los valores dc ácido linoleico obtenidos fueron 5,87\% o para «Microcarpa» Pará; 12,35\% para «Mesocarpa» Soiimóes y 14,21\% para «Macrocarpa» Putumayo. La literatura presenta valores semejantes a éstos; así se tiene que Serruya (1980) encontró 6,27\%; Hammond et al. (1982) 12,5\%; Gómez Da Silva \& Amelotti (1983) 4,8\% y el Ciprona (1986) 15,0\%. 


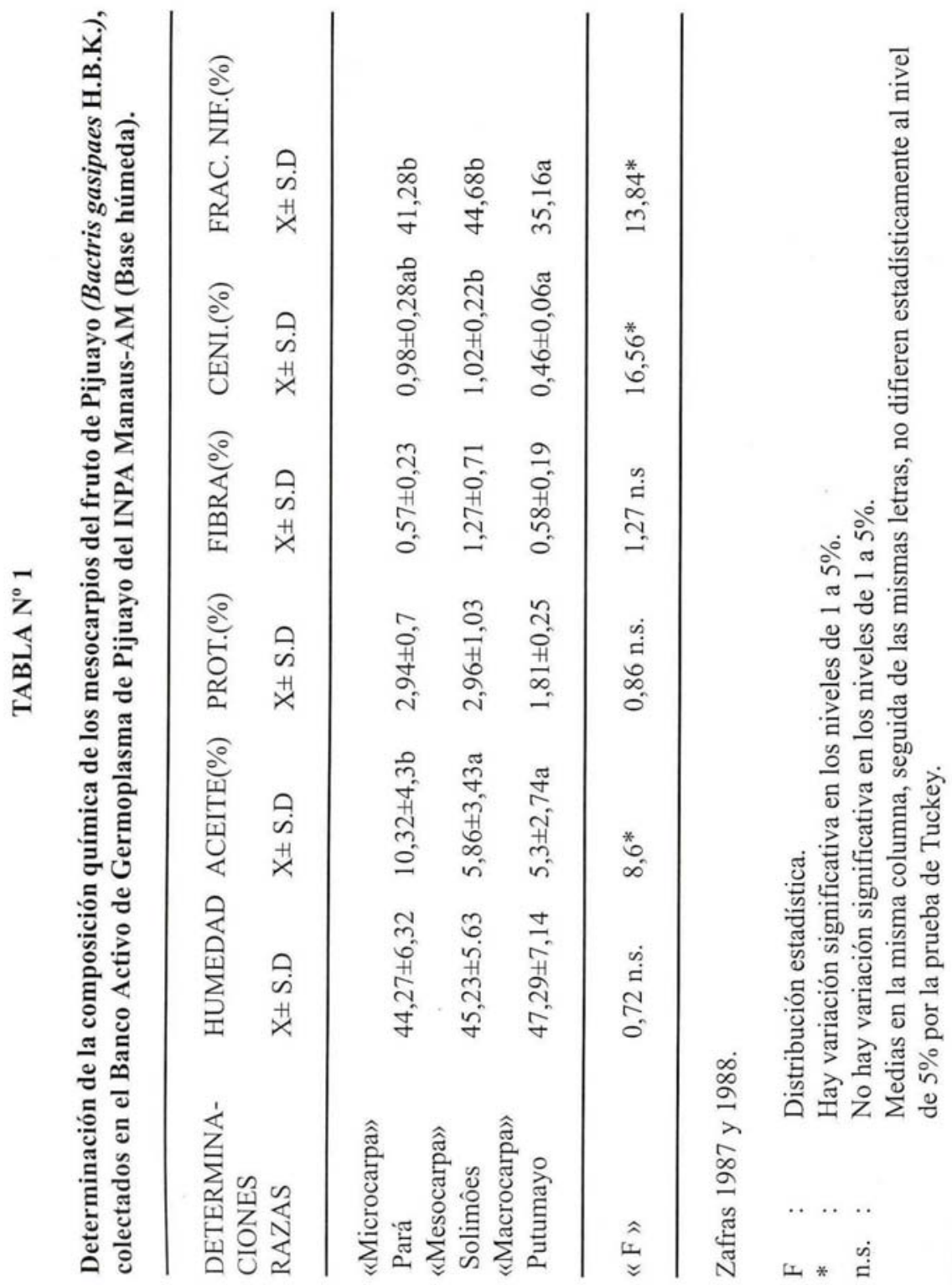




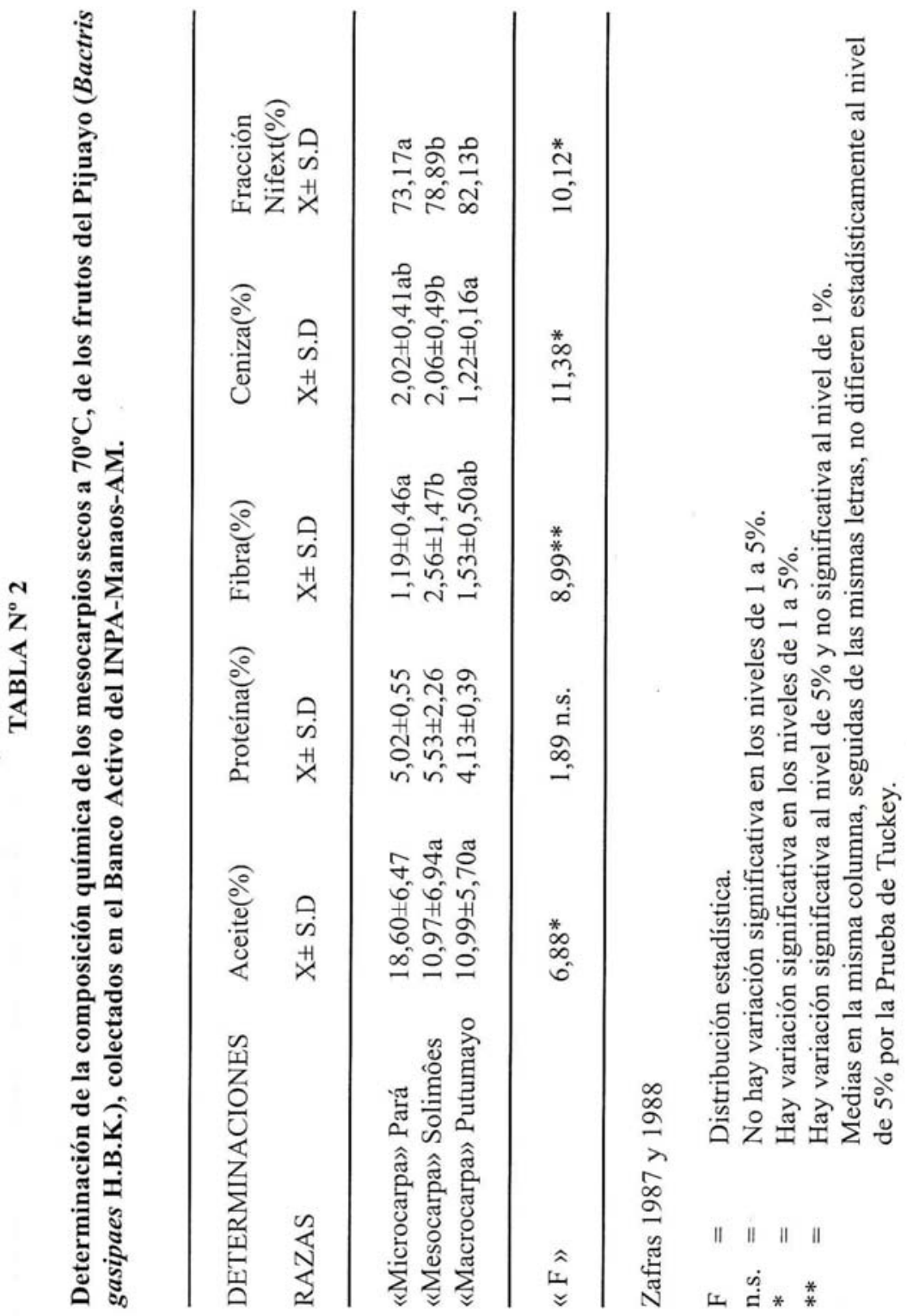




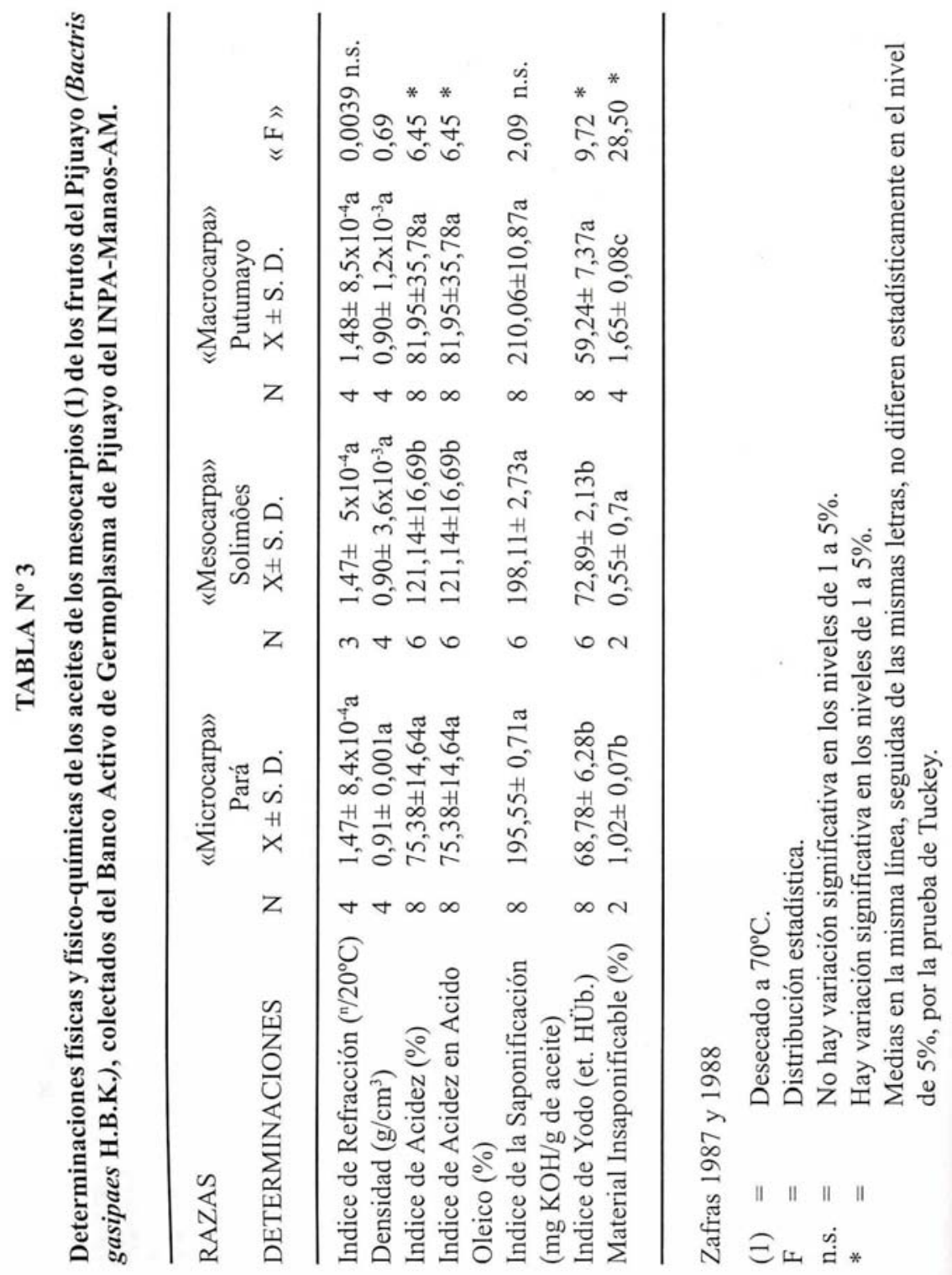




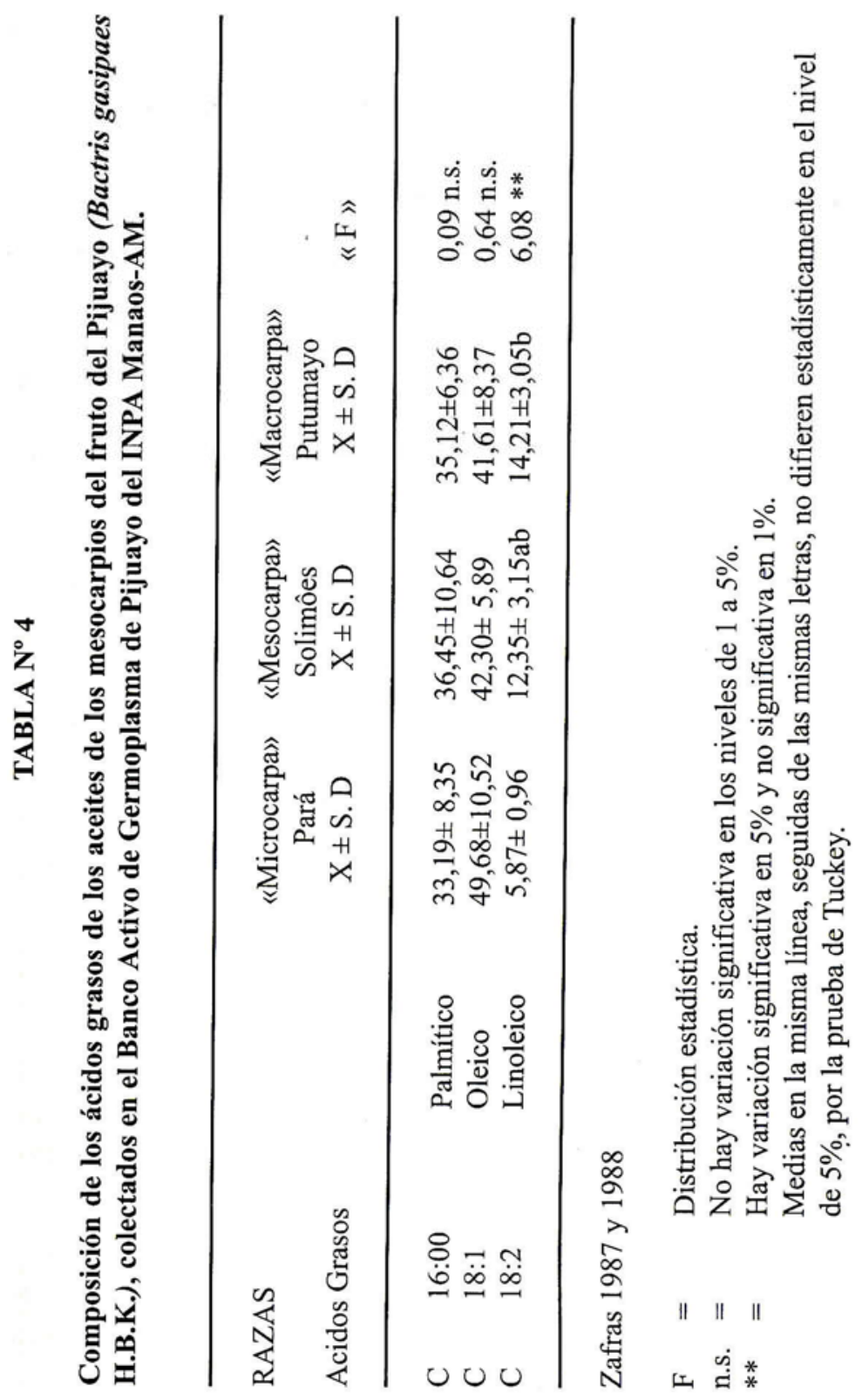




\section{BIBLIOGRAFIA}

AGUIAR, J.; MARINO, H. A.; RABELO, Y S. y SHRIMPTOM, R. 1980. Aspectos nutritivos de algunos frutos de la Amazonía. En: Acta Amazónica 10(4): 755-758.

ARKCOOL, D. B \& AGUIAR, J. P. L. 1984. Peach palm (Bactris gasipaes H.B.K) a new source for vegetable oil palm from the wet tropical. En: J. Sci. Food. Agric. 35:520 .526.

AOAC, (Association of Official Analytical Chemists). 1975. Official Methods of Analysis of the Association of Official Analytica/ Chemists. 20 ed. Washington.

CALZADA, B. J. 1986. Frutas Nativas. Lima: Librería «El Estudiante», 32pp.

CIPRONA, 1986. Aprovechamiento Industrial clel pejibaye (Bactris gasipaes). Universidad de Costa Rica. pp. 64-91.

CLEMENT, C. R. 1988. Domestication of the Pejibaye Palm (Bactris gaszpaes) Past and Present. En: Advances in Economic Botany 6: 155-174.

1993. Pejibaye. En: Clay, J. W. \& Clement C. R. (Eds). Selected species and strategies to enhance income generation from Amazonian forest. Rome: FAO/ FO: MIS/93 /6 Working paper. pp. 92-107.

GOMEZ DA SILVA, W. \& AMELOTTI J, G. 1983. Composizione della sostanza grassa del fmtto di Guilielma speciosa (Pupunha). En: La Rivista Italiana delle Sostanze Grasse. 60: 767-770.

HAMMOND, E. G.; PAN, W. P. \& MORA URPI, J. 1982. Fatty acid composition and gliceride structure of the pejibaye palm (Bactris gasipaes H.B.K.) mesocarp and oil. En: Revta. Biol. Trop., 30 (1): 91.93.

KRONEBERG, A. 1982. Estudio preliminar sobre factores antinutricionales en la harina de pejibaye (Guilielma gasipaes). Tesis para optar el grado de Ingeniero Agrónomo. San José de Costa Rica: Facultad de Agronomía. Universidad de Costa Rica. 70 pp. 
MORA URPI, J. \& CLEMENT, C. R. 1985. Races and populations of Peach palm found in the Amazon basin. En: Clement, C. R. \& Coradin, L. (ed). Final Report; Peach Pal (Bactris gasipaes HiB.K). Germoplasma Bank. USAIND, project report. Costa Rica, pp. 107.141.

SERRUYA, H.; BENJTES, M. H. y ROCHA FJLHO, da G.N. 1980. Analise dos óleos dos frutos de duas palmaceas; Bacaba (Oenocarpus disticus Mart.) e Pupunha (Guilielma speciosa Man.). En: Encontro deprofessionais da Química da Amazônía, 1, Belem. 7pp.

VILLACHICA. H. 1996. Cultivo del Pzjuayo (Bactrisgasipaes Kunth) para palmito en la Amazonía. Lima: Tratado de Cooperación Amazónica. Secretaría Pro Tempore. 153pp.

ZAPATA, A. 1972. Pejibaye palm from the Pacific Coast of Colombia, a detailed chemical analysis. En: Econo. Bot., 26(2): 156-159.

ZAR, Jerrold, H. 1984. Biostatistical Analysis. 2da. ed. New Jersey: Ed. Prentice Hall, Englewood Cliffs. 7117pp.

ZUMBADO, M. E. \& MURILLO, M. G. 1983. Composition and nutritive valve of pejibaye (Bactris gasipaes) and pejibaye meals for animals. En: Rvta. Biol. Trop. 32:51-56. 